\title{
REMARKS ON SECTIONAL CURVATURE OF AN INDEFINITE METRIC ${ }^{1}$
}

\author{
KATSUMI NOMIZU
}

\begin{abstract}
The properties of sectional curvature for an indefinite metric are further studied following the works of R. S. Kulkarni, L. Graves and the author, S. G. Harris, and M. Dajczer and the author.
\end{abstract}

1. Let $M$ be a manifold of dimension $\geqslant 3$ with an indefinite metric of signature $(-, \ldots,+,+, \ldots)$, that is, at least one minus sign and at least two plus signs. A 2-plane $p$ in the tangent space $T_{x}(M)$ is said to be nondegenerate or spacelike or timelike depending on whether the restriction to $p$ of the inner product for the metric is nondegenerate or positive-definite or indefinite, that is, of signature $(-,+)$. Let $K$ be the sectional curvature function defined on the set of all nondegenerate 2-planes $p$. It is a constant function if and only if the curvature tensor $R$ is of the form

$$
R(X, Y) Z=c(\langle Y, Z\rangle X-\langle X, Z\rangle Y)
$$

for all $X, Y, Z$ in $T_{x}(M)$, where $c$ is the constant value of $K$ (see [3]). In this case we say that $M$ has constant sectional curvature at $x$.

It was R. S. Kulkarni [5] who first proved that if $K(p)$ is bounded from below (or from above) for all nondegenerate 2-planes at a point $x$, then $M$ has constant sectional curvature at $x$. It was then remarked in [2 and 4] that if $|K(p)|$ is bounded for all timelike 2-planes $p$ (or for all spacelike 2-planes $p$ ) at $x$, then $M$ has constant sectional curvature at $x$.

The purpose of the present paper is to sharpen these results in the following way.

THEOREM 1. Assume that for each spacelike vector $X$ in $T_{x}(M)$ there is a number $d$ such that $K(p) \geqslant d$ for all nondegenerate 2-planes containing $X$. Then $M$ has constant sectional curvature at $x$. The inequality $K(p) \geqslant d$ can be replaced by $K(p) \leqslant d$.

THEOREM 2. Assume that for each spacelike vector $X$ in $T_{x}(M)$ there is a number $d>0$ such that $|K(p)| \leqslant d$ for all spacelike 2-planes $p$ containing $X$. Then $M$ has constant sectional curvature at $x$. In the assumption, all spacelike 2-planes $p$ can be replaced by all timelike 2-planes $p$ (containing $X$ ).

Received by the editors September 23, 1982.

1980 Mathematics Subject Classification. Primary 53B30; Secondary 15A63.

Key words and phrases. Sectional curvature, indefinite metric, Lorentz metric, timelike 2-planes, spacelike 2-planes.

'Work supported in part by an NSF grant and completed under the program Sonderforschungsbereich “Theoretische Mathematik" (SFB 40) at the University of Bonn. 
2. We shall first prove Theorem 2 by using the following lemma proved in [2].

LEMMA A. Let $V$ be an n-dimensional real vector space with nondegenerate inner product $\langle$,$\rangle of signature (-, \ldots,+, \ldots)$. Let $f$ be a bilinear symmetric function on $V$.

(i) If $f(X, X)=0$ for all null vectors $X$ in $V$, then $f(X, Y)=c\langle X, Y\rangle$ for all $X, Y \in V$.

(ii) If $|f(X, X)| \leqslant d$ for all timelike unit vectors $X$ (or for all spacelike unit vectors $X)$, then

$$
f(X, Y)=c\langle X, Y\rangle \text { for all } X, Y \in V .
$$

Now, according to Theorem la and the subsequent remark in [1], in order to show that $M$ has constant sectional curvature at $x$, it is sufficient to prove that, for any orthonormal vectors $X, Y, Z$ with $\langle X, X\rangle=1$, we have $\langle R(X, Y) Z, X\rangle=0$. This is what we shall now prove under the assumption of Theorem 2. Let $X$ be any spacelike unit vector in $T_{x}(M)$ and suppose $|K(p)| \leqslant d$ for all spacelike 2-planes $p$ containing $X$. Let $V$ be the orthogonal complement of the 1-dimensional subspace spanned by $X$. Note that $V$ inherits an inner product of signature $(-, \ldots,+, \ldots)$. Define a bilinear symmetric function $f$ on $V$ by

$$
f(Y, Z)=\langle R(X, Y) Z, X\rangle \text { for } Y, Z \in V .
$$

If $Y \in V$ is a unit spacelike vector, then $f(Y, Y)$ is nothing but the sectional curvature $K(p)$ of the spacelike 2-plane spanned by $X$ and $Y$. By assumption, $|K(p)| \leqslant d$. In other words, $|f(Y, Y)| \leqslant d$ for all spacelike unit vectors in $V$. By Lemma A, (ii), $f$ is a scalar multiple of the inner product $\langle$,$\rangle . Thus$

$$
\langle R(X, Y) Z, X\rangle=f(Y, Z)=c\langle Y, Z\rangle=0
$$

whenever $Y, Z$ are in $V$ (that is, $\langle X, Y\rangle=\langle X, Z\rangle=0$ ) and $\langle Y, Z\rangle=0$. This proves our assertion and completes the proof of Theorem 2 . In the case of the alternative assumption that $|K(p)| \leqslant d$ for all timelike 2-planes $p$ containing $X$, we simply remark that for any timelike unit vector $Y$ in $V, f(Y, Y)$ is nothing but $-K(p)$, where $p$ is the timelike 2-plane spanned by $X$ and $Y$. Thus $|f(Y, Y)| \leqslant d$ for all timelike unit vectors $Y$ in $V$ so Lemma A, (ii) applies again.

3. We shall now prove Theorem 1 . We shall again prove that $\langle R(X, Y) Z, X\rangle=0$ whenever $X, Y, Z$ are orthonormal and $\langle X, X\rangle=1$. For such $X$, there is a number $d$ such that $K(p) \geqslant d$ for all nondegenerate 2-planes $p$ containing $X$. Again, in the orthogonal complement $V$ of the span of $X$, define $f(Y, Z)=\langle R(X, Y) Z, X\rangle$. If $Y$ is a spacelike unit vector in $V$, then $f(Y, Y)$ is equal to $K(p)$, where $p$ is the spacelike 2-plane spanned by $X$ and $Y$. Thus $f(Y, Y) \geqslant d$. On the other hand, if $Y$ is a timelike unit vector in $V$, then $f(Y, Y)$ is equal to $-K(p)$, where $p$ is the timelike 2-plane spanned by $X$ and $Y$. Thus $-f(Y, Y)=K(p) \geqslant d$, that is, $f(Y, Y) \leqslant-d$. We want to conclude that $f$ is a scalar multiple of the inner product, and this follows from another variation of Lemma $\mathrm{A}$. 
Lemma B. Let $V$ and $f$ be as in Lemma A. Suppose there is a number d such that

$$
f(Y, Y) \geqslant d \text { for all } Y \text { with }\langle Y, Y\rangle=1
$$

and

$$
f(Y, Y) \leqslant-d \text { for all } Y \text { with }\langle Y, Y\rangle=-1 \text {. }
$$

Then

$$
f(X, Y)=c\langle X, Y\rangle \text { for all } X, Y \in V .
$$

Proof. We shall prove that $f(X, X)=0$ for all null vectors $X$ so that Lemma A, (i) applies. Let $X$ be a null vector and choose another null vector $Y$ such that $\langle X, Y\rangle=\frac{1}{2}$. For $t>0,(X+t Y) / \sqrt{t}$ is a unit spacelike vector so that

$$
f\left(\frac{X+t Y}{\sqrt{t}}, \frac{X+t Y}{\sqrt{t}}\right)=\frac{f(X+t Y, X+t Y)}{t} \geqslant d
$$

and hence

$$
f(X, X)+2 t f(X, Y)+t^{2} f(Y, Y) \geqslant t d
$$

By letting $t \rightarrow 0$, we obtain $f(X, X) \geqslant 0$.

Now for $t<0,(X+t Y) / \sqrt{-t}$ is a unit timelike vector so that

$$
f\left(\frac{X+t Y}{\sqrt{-t}}, \frac{X+t Y}{\sqrt{-t}}\right)=\frac{f(X+t Y, X+t Y)}{-t} \leqslant-d
$$

and hence

$$
f(X, X)+2 t f(X, Y)+t^{2} f(Y, Y) \leqslant t d .
$$

By letting $t \rightarrow 0$, we obtain $f(X, X) \leqslant 0$. This proves $f(X, X)=0$, as we wanted. Lemma $B$ is thus proved.

For the alternative assumption in Theorem 1, the proof is similar; in the assumptions of Lemma $\mathrm{B}$, the inequalities can be reversed. This completes the proof of Theorem 1.

REMARK. Theorems 1 and 2 are formulated so that they are valid when $M$ is a Lorentzian manifold, that is, the metric has signature $(-,+, \ldots,+)$. If the signature is $(-,-, \ldots,+, \ldots)$, we may state obvious variations of Theorems 1 and 2 . For example, if, for each timelike vector $X,|K(p)|$ is bounded for all timelike 2-planes containing $X$, then $M$ has constant sectional curvature. This statement is not true if $M$ is a Lorentzian manifold.

\section{REFERENCES}

1. M. Dajczer and K. Nomizu, On sectional curvature of indefnite metrics. II, Math. Ann. 247 (1980), 279-282.

2. $25-30$. , On the boundedness of Ricci curvature of an indefinite metric, Bol. Soc. Brasil. Mat. 11 (1980),

3. L. Graves and K. Nomizu, On sectional curvature of indefinite metrics, Math. Ann. 232 (1978), 267-272. 
4. S. G. Harris, A triangle comparison theorem for Lorentz manifolds, Indiana Univ. Math. J. 31 (1982), 289-308.

5. R. S. Kulkarni, The values of sectional curvature in indefinite metrics, Comment Math. Helv. 54 (1979), 173-176.

Department of Mathematics, Brown University, Providence, Rhode Island 02912(Current address)

Max-Planck-Institut fUr Mathematik, Gottfried-Claren-Strasse 26, 5300 Bonn 3, Federal REPUBLIC OF GERMANY 